\title{
Os Odontoideum: A Comprehensive Clinical and Surgical Review
}

Fareed Jumah ${ }^{1}$, Saja Alkhdour ${ }^{1}$, Shaden Mansour ${ }^{1}$, Puhan $\mathrm{He}^{2}$, Ali Hroub ${ }^{1}$, Nimer Adeeb ${ }^{3}$, Rimal H. Dossani ${ }^{3}$, Martin M. Mortazavi ${ }^{4}$, R. Shane Tubbs ${ }^{5}$, Anil Nanda ${ }^{3}$

1. School of Medicine, An-najah National University Hospital, Nablus, Palestine 2. Harvard School of Dental Medicine, Harvard University 3. Department of Neurosurgery, Louisiana State University, Shreveport, LA. 4. California Institute of Neuroscience, Los Robles Hospital and Medical Center 5. Neurosurgery, Seattle Science Foundation

$\square$ Corresponding author: Puhan He, puhanhe@gmail.com

Disclosures can be found in Additional Information at the end of the article

\section{Abstract}

Os odontoideum $(\mathrm{OO})$ is a rare anomaly of the odontoid process first described by Giacomini in 1886. There is considerable debate about the origin of this anomaly, whether congenital or acquired, though a growing body of evidence favors the latter.

Using PubMed, we reviewed the literature on $O 0$ with regards to its etiology, clinical presentations, diagnostic modalities, and management. Manuscripts cited in reviews were also searched manually.

Because the medical literature on this condition is limited, our understanding of the natural history and management of $\mathrm{OO}$ is still vague. The management guidelines for asymptomatic $\mathrm{OO}$ are preliminary. Therefore, we need more large-center studies to investigate this condition further.

Categories: Radiology, Neurosurgery, Orthopedics

Keywords: os odontoideum, odontoid, dens, c2, fracture, spine, cervical, review

\section{Introduction And Background}

Os odontoideum $(\mathrm{OO})$ is a congenital anomaly of the second cervical vertebra (axis), defined as a smooth, independent ossicle of variable size and shape separated from the base of a shortened odontoid process by an obvious gap, with no osseous connection to the body of C2 [1]. OO can be classified into two anatomical types, orthotopic and dystopic [2]. (Figure 1) An orthotropic OO lies in the normal position on the odontoid process, moving with the atlas anterior arch, while the dystopic morphology describes an ossicle fused to the basion.

Since the first description by Giacomini in 1886, whether the etiology of OO is congenital or traumatic has remained a subject of controversy. However, regardless of its etiology, $\mathrm{OO}$ remains a major compromise to atlanto-axial stability, even under physiological loads in some individuals, placing the spinal cord and the vertebral artery at a significant risk of injury. Moreover, the spectrum of clinical presentations is striking, ranging from mild neurological symptoms to paralysis. 


\section{Cureus}

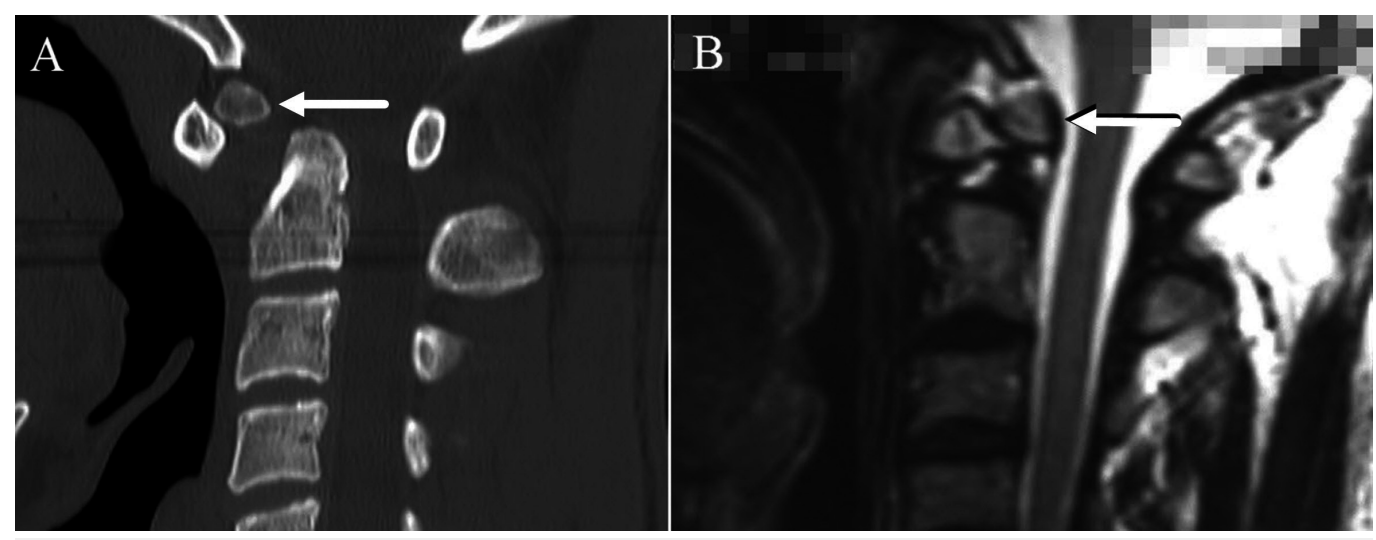

FIGURE 1: Spine imaging showing the two anatomic subtypes of os odontoideum: dystopic (Panel A) and orthotopic (Panel B)

\section{Review}

\section{Embryology}

Defining the anatomy and embryology of the odontoid is crucial for understanding the etiology of $\mathrm{OO}$ and for reducing the rates of false-positive diagnoses, especially in the pediatric population. Early in development, the fourth occipital sclerotome forms the apex of the odontoid, which is called the ossiculum terminal or the apical odontoid epiphysis [3], while the first and second cervical sclerotomes contribute to the odontoid and axis bodies, respectively [4-5]. After birth, the odontoid has an epiphyseal growth plate separating the first and second cervical sclerotomes known as the neurocentral synchondrosis, which lies below the level of the superior articular facets of the axis and is usually visible in children younger than three or four years but disappears by eight years of age [6]. The odontoid has a different blood supply from the rest of the spine. The neurocentral synchondrosis precludes a rostral vascular supply by the anterior and posterior branches of the vertebral artery. As a result, the odontoid process depends significantly on a terminal descending supply superiorly, called the apical arcade. This relative deficiency of the odontoid blood supply places it at a significant risk of ischemia and necrosis. The blood supply of the odontoid process comes from two sources and is different from the rest of the spine. The vertebral artery gives off posterior ascending arteries at the level of C3, which supply deep penetrating branches as they pass anterior and posterior to the bodies of the axis and the odontoid process, eventually anastomosing with the apical arcade.

Furthermore, the anterior ascending arteries and the apical arcade anastomose with branches from the carotid arteries through the base of the skull and the alar ligaments. This arterial apparatus is crucial early in life since no vessels pass through the transient epiphyseal plate between the odontoid process and the axis. More importantly, the relatively fixed position of the dens as the atlas rotates prevents sufficient vascularization by the anterior and posterior branches of the vertebral arteries. Consequently, the odontoid process depends significantly on a terminal descending supply superiorly (the apical arcade). This relative deficiency of the odontoid blood supply renders it particularly vulnerable to ischemia and necrosis, especially in traumatic events. Moreover, the blood supply of the odontoid process may be unstable because the blood vessels traverse closely alongside the odontoid process and, hence, can be easily obstructed. Such an obstruction leads to ischemia that may contribute to poor fracture healing and callus formation.

\section{Etiology}


The etiology of OO remains controversial, although a growing body of evidence favors the traumatic over the congenital hypothesis. According to this hypothesis, OO results from a failure of the dens to fuse with the body of the axis during embryonic development [2] or of the secondary ossification center at the apex of the dens to fuse with its main part [2]. Another possible explanation allowed by this hypothesis is the failure of proper caudal migration of the dens during development [7]. This condition has been described in identical twins [7] and in families, suggesting an autosomal dominant pattern [8]. The congenital etiology is further supported by the association of OO with many congenital syndromes and malformations such as bipartite atlas [9], Morquio's disease [10], the Klippel-Feil syndrome [11], multiple epiphyseal dysplasia [10], achondroplasia [12], the Larson syndrome [13], the Wolcott-Rallison syndrome [14], and chondrodystrophia calcificans [15]. Authors supporting the congenital hypothesis have proposed that trauma per se could not lead to OO formation. Instead, a traumatic event could increase instability due to soft tissue injury, consequently unmasking a pre-existing OO.

The post-traumatic or acquired hypothesis originated mainly from the work of Fielding and Griffin [16], who proposed that OO forms after an unrecognized fracture to the odontoid with the subsequent contraction of the apical and alar ligaments, the distraction of the fractured fragment, and the severing of blood supply, leading to the formation of an ossicle, the OO. Several other studies have supported the post-traumatic etiology [17-19,40]. Proponents of this hypothesis argue that $\mathrm{OO}$ is most commonly located at the base of the dens and not at the synchondrosis, where a congenital fusion failure would be expected to occur. In addition, Verska and Anderson [20] presented a case report of a post-traumatic OO in one identical twin, the other twin having a completely normal cervical radiograph with no history of trauma, which argues against a congenital etiology. Case report investigations of children with OO indicate a vascular etiology, such as post-traumatic avascular necrosis [21], or post-traumatic blood supply blockage to the proximal odontoid with osseous absorption, where the ossiculum terminale continues to receive a normal blood supply, forming the OO [22]. Nevertheless, a combination of both etiologies is supported by the argument that deficient ossification of the odontoid and hyperlaxity of ligaments in the previously mentioned congenital syndromes predispose individuals to traumatic OO [23]. However, regardless of the etiology, diagnosis and management remain the same [24].

\section{Clinical Presentation and Complications}

Since the first description of OO, researchers have enriched the literature with numerous case reports and series related to the condition. The exact incidence and prevalence remain unclear because of the rarity of this anomaly and its silent course in many individuals [25]. However, while looking for anatomical variations of the odontoid process, Perdikakis et al. found that 0.7 percent of patients examined for probable cervical spine pathology had OO, and Sankar et al. detected it in 3.1 percent of children with abnormal cervical radiographs [26-27]. Although the etiology, natural history, and need for surgery remain controversial, clinical presentation among patients with $\mathrm{OO}$ can be classified into four main categories: incidental finding in asymptomatic patients, local symptoms, cervical myelopathic symptoms and signs, and symptoms related to vertebrobasilar ischemia [28]. This wide range of manifestations can be attributed to multiple factors, such as the slow progression of atlanto-axial instability and irritation caused by an OO [16], the anatomical type of OO (dystopic vs. orthotopic) [29], and the radiographic morphology of the atlanto-axial joint, the round type being associated with more severe myelopathic manifestations than either the cone or blunt-tooth types [30].

The onset of symptoms is noticeably related to traumatic events, including minor ones, and patients are frequently diagnosed during adolescence and early adulthood [31-32]. Neck pain and stiffness, shoulder pain, torticollis, and occipital headaches are the most common local symptoms in patients with 00 [31-32]. Several atypical symptoms have also been reported. For example, Zussman et al. presented a case of chronic posterior thoracic pain refractory to 
symptomatic treatment, which was later diagnosed with cervical instability secondary to $\mathrm{OO}$ [33]. However, because of the high prevalence of symptoms such as headaches and neck pain in the general population, their association with $\mathrm{OO}$ is difficult to establish.

Patients with myelopathy can present with transient or progressive symptoms related to the site and degree of compression [25,32]. The literature documents a wide range of such symptoms, including weakness, numbness, and paresthesias [2], the central cord syndrome, the Brown-Séquard syndrome [34], Lhermitte's phenomenon [35], the central hypoventilation syndrome (Ondine's curse) [29], and cardiorespiratory arrest and sudden death [36]. One case report described the association of $\mathrm{OO}$ with the ossification of the posterior atlanto-axial membrane in an adult patient with severe myelopathy and assumed that the ossification, which is a rare cause of myelopathy, was a consequence of chronic mechanical stress on the ligament [37].

OO has also been implicated in the development of cerebellar infarction [38]. Threedimensional (3D) computed tomographic (CT) angiography showed that atlanto-axial dislocation resulted in the irregular narrowing of the vertebral artery, so the endothelium there is a likely focus for embolus formation [39].

\section{Differential Diagnosis of OS}

Based on history and physical exam, the clinical manifestations of OO may resemble a myriad of other conditions, most notably degenerative disc disease of the cervical spine, cervical spondylosis, and Grade II mechanical neck pain, or atlantoaxial subluxation (e.g., due to rheumatoid arthritis).

On the other hand, the radiographic differential diagnosis of $\mathrm{OO}$ is limited. The main diagnosis to consider is an acute fracture of the odontoid process. $\mathrm{OO}$ can be distinguished by the smooth surface of the ossicle and the underlying body peg of $\mathrm{C} 2$, the absence of a recent history of trauma, and the possible sclerosis and hypertrophy of the anterior tubercle of the atlas. A persistent ossiculum terminale is another differential diagnosis that may be confused with $0 O$. It is caused by the nonunion of the apex at the secondary ossification center. However, it is rarely associated with $\mathrm{C} 1-\mathrm{C} 2$ instability and, consequently, does not require surgical correction.

\section{Radiological Findings and Imaging Modalities in the Diagnosis and Assessment of $\mathrm{OO}$}

Broadly speaking, OO can be clearly visualized using plain radiographs with the open mouth, anteroposterior, and lateral views. In addition, plain dynamic lateral radiographs (performed in flexion and extension) can further evaluate atlanto-axial instability. Nevertheless, the sensitivity and specificity of these imaging modalities have not been studied [25]. On the other hand, CT scans, CT scans with angiography, and magnetic resonance imaging (MRI) scans are important for a better illustration of osseous abnormalities, the arrangement of vertebral arteries, and spinal cord compression and pathology, respectively. Such illustration is mandatory for identifying the exact causes of the patients' symptoms and planning for surgery [25]. Furthermore, Hughes et al. recommended the use of kinematic MRI in diagnosing OO, given the advantage of directly visualizing the motions of joint components and the surrounding soft tissues [41]. However, an initial examination of patients with myelopathy using a conventional MRI scan can occasionally lead to a misdiagnosis of chronic cervical spine instability secondary to $\mathrm{OO}$ as an intramedullary spinal cord tumor [42].

In attempts to correlate symptomatic status with accurate parameters, several indicators for assessing instability have been suggested. The most commonly used ones are the direction of 
atlanto-axial instability, whether anterior (most common), posterior, or multidirectional [43], the space available for the spinal cord (using $13 \mathrm{~mm}$ as a cut-off) [44], and the instability index ( more than 40 percent being significant) [45]. Nonetheless, many researchers have concluded that such parameters cannot reflect the true degree of instability [46]. Moreover, using MRI scans, Chang et al. suggested that myelopathy in patients with $\mathrm{OO}$ is a result of retrodental cystic and fibrocartilaginous masses rather than atlanto-axial instability [47].

This considerable concern about suitable parameters is mainly attributable to the need to set clear guidelines for managing patients with 00 , especially those who are asymptomatic.

\section{Cadaveric Findings}

In the first study of its kind, Sardi et al. report the cadaveric findings of a 72-year-old male with OO but no history of craniocervical instability. The cause of death was unknown. On gross examination, the specimen did not display any arthritic degeneration. The proximal portion of the odontoid process was observed as a clear continuation of the axis body, measuring $12.96 \mathrm{x}$ 11.16 x $11.93 \mathrm{~mm}$ (L x D x W). However, the distal fragment measured $10.03 \times 6.06 \times 4.03 \mathrm{~mm}$ (L $\mathrm{x} \mathrm{D} \times \mathrm{W}$ ) and was separated from the rest of the odontoid process by a small gap. The OO was orthotopic, quite mobile, and in contact with the odontoid process with evidence of remodeling, but no articular cartilage was observed. Interestingly, however, the CT measurements of the $\mathrm{OO}$ were almost half as large $(5.15 \times 5.39$ × $3.84 \mathrm{~mm}(\mathrm{~L} \mathrm{x} \mathrm{W} \mathrm{x} \mathrm{D)})$.

\section{Management of $\mathrm{OO}$}

The management guidelines for $\mathrm{OO}$ remain vague because the evidence in the literature is limited, especially since the condition is rare and its natural history poorly understood. There is general agreement that patients with symptomatic $\mathrm{OO}$ (e.g. cervical myelopathy) should be treated surgically. However, debate still continues regarding the treatment of asymptomatic oO.

Dai et al. noted that five cases of asymptomatic OO that were managed conservatively remained stable at follow-up more than one year later [48]. Others have found no difference in outcome between the conservative and surgical treatment of patients with asymptomatic $\mathrm{OO}$ $[32,49]$. Therefore, most authors believe a conservative approach is adequate if $\mathrm{OO}$ is asymptomatic, while clinical and radiological follow-ups are used to monitor for radiographic instability or significant symptoms $[25,43,46,50]$. However, a subset of asymptomatic patients is thought to be at risk for deterioration, so these patients should be considered for prophylactic spinal fusion. For example, Spierings and Braakman [32] found that asymptomatic OO patients with a minimal sagittal diameter of less than $13 \mathrm{~mm}$ had the greatest risk of spinal cord injury. Other asymptomatic patients who are potential candidates for surgical intervention are young, have favorable anatomy, and show radiographic evidence of atlanto-axial instability on flexion extension X-rays [43].

On the other hand, because evidence concerning the long-term natural course of untreated $\mathrm{OO}$ is limited, some authors [31] believe that all asymptomatic patients should undergo C1-C2 fusion to avert neurological complications, even those with a 'stable' OO. This can be better appreciated when we consider reports in the literature on sudden death [36,48], significant neurological complications [10] following minor injuries in previously undiagnosed $\mathrm{OO}$, and patients who suffer late neurological deterioration [31]. Moreover, these patients are at increased risk of neurological injury during the flexion and extension of the neck during intubation and patient positioning [47]. For those reasons, Class III medical evidence in the literature suggests that prophylactic $\mathrm{C} 1-\mathrm{C} 2$ fusion is meritorious in such cases [50].

It is worth mentioning that surgeons have attempted to define radiographic risk factors that 
predict a high risk of spinal cord injury in asymptomatic patients. Atlanto-axial instability has been defined as more than $3 \mathrm{~mm}$ of motion at C1-C2 on flexion-extension films [2]. However, neither cervical flexion-extension X-rays nor the degree of atlanto-axial subluxation on static imaging (CT, MRI, and plain films) has been shown to correlate with neurological status [32]. Nevertheless, some authors found that a sagittal spinal canal diameter less than $13 \mathrm{~mm}$ was strongly associated with myelopathy [32], while patients with transient or progressive myelopathy had an instability index of more than 40 percent or a sagittal plane rotation more than 20 larger than in patients without neurological symptoms [44]. The "round" type of OO morphology correlates more strongly with myelopathy [30]. Age is another important factor in managing asymptomatic OO. Children, for example, are more prone to falls from day-to-day activities, so they are more likely to need protection against the inherently unstable OO [43]. Moreover, although daily activities can be modified to reduce the risk of injury (e.g., contact sports), incidental injuries and road traffic accidents remain a risk factor shared among all age groups.

Regarding the management of symptomatic OO, the literature still lacks clear, high-quality Class I or II evidence, and current management guidelines all come from Class III evidence (case reports and case series). The most common technique is posterior C1-C2 fixation and fusion, although other successful approaches have been used [50].

\section{Conclusions}

Our knowledge of this rare and poorly understood condition continues to evolve. Physicians should be familiar with $\mathrm{OO}$ and its wide array of manifestations and provide appropriate management that can prevent potentially catastrophic consequences. While there is agreement about the decision to operate on symptomatic OO, patients with asymptomatic OO should be approached more carefully. Physicians should educate them about the unpredictable course of their condition and discuss the available treatment options while keeping in mind that surgery does not come without risks. Given the whole view, the latest recommendation regarding the management of asymptomatic patients suggests either regular clinical and radiographic followup or posterior internal fixation and fusion of C1-C2. The natural history of OO still remains vague. More studies, such as a multicenter natural history study, are needed to improve our understanding of this condition and to produce high-quality evidence for its management.

\section{Additional Information \\ Disclosures}

Conflicts of interest: In compliance with the ICMJE uniform disclosure form, all authors declare the following: Payment/services info: All authors have declared that no financial support was received from any organization for the submitted work. Financial relationships: All authors have declared that they have no financial relationships at present or within the previous three years with any organizations that might have an interest in the submitted work. Other relationships: All authors have declared that there are no other relationships or activities that could appear to have influenced the submitted work.

\section{References}

1. Visocchi M, Fernandez E, Ciampini A, et al.: Reducible and irreducible os odontoideum in childhood treated with posterior wiring, instrumentation and fusion. Past or present?. Acta Neurochir. 2009, 151:1265-1274.

2. Fielding JW, Hensinger RN, Hawkins RJ: Os Odontoideum. J Bone Joint Surg Am. 1980, 62:376-383.

3. Viswanathan A, Whitehead WE, Luerssen TG, et al.: "Orthotopic" ossiculum terminale persistens and atlantoaxial instability in a child less than 12 years of age: a case report and 
review of the literature. Cases J. 2009, 2:8530. 10.4076/1757-1626-2-8530

4. Currarino G: Segmentation defect in the midodontoid process and its possible relationship to the congenital type of os odontoideum. Pediatr Radiol. 2002, 32:34-40. 10.1007/s00247-0010579-1

5. Menezes AH: Craniocervical developmental anatomy and its implications. ChNS. 2008, 24:1109-1122. 10.1007/s00381-008-0600-1

6. Menezes AH: Congenital and acquired abnormalities of the craniovertebral junction . Neurological Surger. Youmans J (ed): Saunders Publishers, Philadelphia; 1995.

7. Kirlew KA, Hathout GM, Reiter SD, et al.: Os odontoideum in identical twins: perspectives on etiology. Skeletal Radiol. 1993, 22:525-527. 10.1007/BF00209102

8. Wang S, Wang C: Familial dystopic os odontoideum: a report of three cases . J Bone Joint Surg Am. 2011, 93:44. 10.2106/jbjs.j.01018

9. Osti M, Philipp H, Meusburger B, et al.: Os odontoideum with bipartite atlas and segmental instability: a case report. Eur Spine J. 2006, 15:564-567. 10.1007/s00586-005-0017-4

10. Stevens JM, Chong WK, Barber C, et al.: A new appraisal of abnormalities of the odontoid process associated with atlanto-axial subluxation and neurological disability. Brain. 1994, 1:133-148. 10.1093/brain/117.1.133

11. Morgan MK, Onofrio BM, Bender CE: Familial os odontoideum. Case report. J Neurosurg. 1989, 70:636-639. 10.3171/jns.1989.70.4.0636

12. Yukata K, Katoh S, Sairyo K, et al.: Os odontoideum in achondroplasia: a case report . J Pediatr Orthop. 2008, 17:103-105. 10.1097/BPB.0b013e3282f54a55

13. Kornreich L, Horev G, Schwarz M, et al.: Laron syndrome abnormalities: spinal stenosis, os odontoideum, degenerative changes of the atlanto-odontoid joint, and small oropharynx. Am J Neuroradiol. 2002, 23:625-631.

14. Dias RP, Buchanan CR, Thomas N, et al.: Os odontoideum in wolcott-rallison syndrome: a case series of 4 patients. Orphanet J. Rare Dis. 2016, 11:14. 10.1186/s13023-016-0397-Z

15. Bethem D: Os odontoideum in chondrodystrophia calcificans congenita. A case report . J Bone Joint Surg. 1982, 64:1385-1386.

16. Fielding JW, Griffin PP: Os odontoideum: an acquired lesion. J Bone Joint Surg. 1974, 56:187190.

17. Ricciardi JE, Kaufer H, Louis DS: Acquired os odontoideum following acute ligament injury. Report of a case. J Bone Joint Surg. 1976, 58:410-412.

18. Schuler TC, Kurz L, Thompson DE, et al.: Natural history of os odontoideum. J Pediatr Orthop. 1991, 11:222-225.

19. Stillwell WT, Fielding JW: Acquired os odontoideum. A case report. Clinical orthopaedics and related research. 1978, 135:71-73.

20. Verska JM, Anderson PA: Os odontoideum. A case report of one identical twin . Spine. 1997, 22:706-709.

21. Sakaida H, Waga S, Kojima T, et al.: Os odontoideum associated with hypertrophic ossiculum terminale. Case report. J Neurosurg. 2001, 94:140-144. 10.3171/spi.2001.94.1.0140

22. Hammerstein J, Russo S, Easton K: Atlantoaxial dislocation in a child secondary to a displaced chondrum terminale. A case report. J Bone Joint Surg. 2007, 89:413-417.

23. Wang S, Wang C: Acquired os odontoideum: a case report and literature review . ChNS. 2012, 28:315-319. 10.1007/s00381-011-1642-3

24. Nguyen JC, Pollock AN: Os odontoideum. Pediatr Emerg Care. 2015, 31:225-227. 10.1097/pec.0000000000000411

25. Arvin B, Fournier-Gosselin MP, Fehlings MG: Os odontoideum: etiology and surgical management. Neurosurgery. 2010, 66:22-31. 10.1227/01.neu.0000366113.15248.07

26. Sankar WN, Wills BP, Dormans JP, Drummond DS: Os odontoideum revisited: the case for a multifactorial etiology. Spine. 2006, 31:979-984. 10.1097/01.brs.0000214935.70868.1c

27. Perdikakis E, Skoulikaris N: The odontoid process: various configuration types in MR examinations. Eur Spine J. 2014, 23:1077-1083. 10.1007/s00586-013-3135-4

28. Tejada Meza H, Modrego Pardo P, Gazulla Abio J: Cervical myelopathy as the initial manifestation of os odontoideum. Neurologia. 2016, 31:278-279. 10.1016/j.nrl.2014.06.006

29. Campbell E, Brown J: Case report of os odontoideum causing Ondine's curse . Br J Neurosurg. 2013, 27:836-837. 10.3109/02688697.2013.795520

30. Matsui H, Imada K, Tsuji H: Radiographic classification of Os odontoideum and its clinical significance. Spine. 1997, 22:1706-1709. 
31. Klimo P Jr, Kan P, Rao G, et al.: Os odontoideum: presentation, diagnosis, and treatment in a series of 78 patients. J Neurosurg Spine. 2008, 9:332-342. 10.3171/SPI.2008.9.10.332

32. Spierings EL, Braakman R: The management of os odontoideum. Analysis of 37 cases . J Bone Joint Surg Br. 1982, 64:422-428.

33. Zussman BM, Saldua NS, Harrop JS: Cervical instability presenting as thoracic pain: case report and literature review. J Spinal Cord Med. 2012, 35:125-127.

10.1179/1079026812z.00000000023

34. Zhang Z, Wang H, Liu C: Acute traumatic cervical cord injury in pediatric patients with os odontoideum: a series of 6 patients. World Neurosurg. 2015, 83:1180-1181.

10.1016/j.wneu.2014.12.036

35. Brecknell JE, Malham GM: Os odontoideum: report of three cases . J Clin Neurosci. 2008, 15:295-301. 10.1016/j.jocn.2006.07.022

36. Michaels L, Prevost MJ, Crang DF: Pathological changes in a case of os odontoideum (separate odontoid process). J Bone Joint Surg Am. 1969, 51:965-972.

37. Ohya J, Chikuda H, Sugita S, et al.: Ossification of the posterior atlantoaxial membrane associated with an os odontoideum: a case report. J Orthop Surg. 2011, 19:392-394. 10.1177/230949901101900330

38. Sasaki H, Itoh $\mathrm{T}$, Takei $\mathrm{H}$, et al.: Os odontoideum with cerebellar infarction: a case report . Spine. 2000, 25:1178-1181.

39. Fukuda M, Aiba T, Akiyama K, et al.: Cerebellar infarction secondary to os odontoideum. J Clin Neurosci. 2003, 10:625-626. 10.1016/S0967-5868(03)00131-0

40. Visocchi M, Di Rocco C: Os odontoideum syndrome: pathogenesis, clinical patterns and indication for surgical strategies in childhood. Adv Tech Stand Neurosurg. 2014, 40:273-293. 10.1007/978-3-319-01065-6_9

41. Hughes TB Jr, Richman JD, Rothfus WE: Diagnosis of Os odontoideum using kinematic magnetic resonance imaging. A case report. Spine. 1999, 24:715-718.

42. Gigante PR, Feldstein NA, Anderson RC: C1-2 instability from os odontoideum mimicking intramedullary spinal cord tumor. J Neurosurg Pediatr. 2011, 8:363-366.

10.3171/2011.7.peds1186

43. Klimo P, Jr., Coon V, Brockmeyer D: Incidental os odontoideum: current management strategies. Neurosurg Focus. 2011, 31:10. 10.3171/2011.9.focus11227

44. Watanabe M, Toyama Y, Fujimura Y: Atlantoaxial instability in os odontoideum with myelopathy. Spine. 1996, 21:1435-1439.

45. Abe H, Tsuru M, Mitsumori K: [Atlanto-axial dislocation--instability index and indications for surgery (author's transl)]. No Shinkei Geka. 1976, 4:57-72.

46. Menezes AH: Pathogenesis, dynamics, and management of os odontoideum . Neurosurgical focus. 1999, 6:2.

47. Chang H, Park JB, Kim KW, et al.: Retro-dental reactive lesions related to development of myelopathy in patients with atlantoaxial instability secondary to Os odontoideum. Spine. 2000, 25:2777-2783.

48. Dai L, Yuan W, Ni B, et al.: Os odontoideum: etiology, diagnosis, and management. World Neurosurg. 2000, 53:106-109. 10.1016/S0090-3019(99)00184-6

49. Wilson JR, Dettori JR, Vanalstyne EM, et al.: Addressing the challenges and controversies of managing os odontoideum: results of a systematic review. Evid Based Spine Care J. 2010, 1:67-74. 10.1055/s-0028-1100896

50. Rozzelle CJ, Aarabi B, Dhall SS, et al.: Os odontoideum. Neurosurgery. 2013, 72:159-169. 10.1227/NEU.0b013e318276ee69 